PROCEEDINGS OF THE AMERICAN MATHEMATICAL SOCIETY

Volume 124, Number 6, June 1996

\title{
DESCENT OF THE CANONICAL MODULE IN RINGS WITH THE APPROXIMATION PROPERTY
}

\author{
CHRISTEL ROTTHAUS
}

(Communicated by Wolmer V. Vasconcelos)

\begin{abstract}
Let $(R, m)$ be a local Noetherian Cohen-Macaulay ring with the approximation property. We show that $R$ admits a canonical module.
\end{abstract}

\section{INTRODUCTION}

In $[H], V$. Hinich shows that rings with the approximation property admit a dualizing complex. The proof involves some deep and possibly less standard results from homological algebra. It is well known that for local, Noetherian, CohenMacaulay rings the existence of a dualizing complex is equivalent to the existence of a canonical module. The purpose of this paper is to present an elementary proof for the existence of a canonical module in Cohen-Macaulay rings with the approximation property.

The proof makes use of an easy observation which is called complete induction. Complete induction imitates the often easier - but special - situation where the completion of a local ring $R$ can be obtained from $R$ by completing $R$ with respect to a principal ideal. This method requires that the class of rings under consideration be closed under ideal-adic completions. Unfortunately, it is not known yet if the class of rings with the approximation property satisfies this requirement. Thus we are forced to restrict our discussion to a certain class of rings with the approximation property - the, so called, rings with the complete approximation property.

Rings with the complete approximation property are defined as local Noetherian rings $R$ for which any ideal-adic completion of $R$ possesses the approximation property. M. Artin conjectured that excellent, Henselian rings have the approximation property. It is an easy Corollary from Artin's conjecture that rings with the approximation property also admit the complete approximation property. Since Artin's conjecture already has been shown for a huge class of excellent, Henselian rings, we do not believe that restricting consideration of the class of approximation rings to the class of complete approximation rings presents a serious limitation.

Received by the editors September 16, 1994 and, in revised form, December 14, 1994.

1991 Mathematics Subject Classification. Primary 13B35, 13B40, 13D45, 13F40, 13J15.

Key words and phrases. Canonical modules, dualizing complexes, Cohen-Macaulay rings, approximation property, excellent local Henselian rings.

The author gratefully acknowledges partial support from the National Science Foundation.

(c)1996 American Mathematical Society 


\section{Preliminaries}

In the following we use the notation and definitions of Matsumura's book $[\mathrm{M}]$. If $(R, m)$ is a local Noetherian ring, its $m$-adic completion is denoted by $\hat{R}$ and referred to as "the" completion of $R$. Additionally, we consider completions of $R$ with respect to $I$-adic topologies of $R$ where $I \subseteq R$ is an ideal. These are denoted by $(R, I)^{\wedge}$ or $R^{*}$, if the reference to the ideal $I$ is obvious.

In this paper we are concerned with the proof that a certain class of local Noetherian rings inherits the canonical module from its completion. The main technique of the proof is complete induction which is explicitly described in $[R]$. Complete induction is based on the following.

2.1. Proposition. Let $(R, m)$ be a local Noetherian ring which satisfies some property $\mathcal{P}$ and suppose that its completion $\hat{R}$ fails to satisfy $\mathcal{P}$. Then there is an ideal $I \subseteq R$ such that:

(a) $R^{*}=(R, I)^{\wedge}$ satisfies $\mathcal{P}$.

(b) For every element $s^{*} \in m R^{*}-I R^{*}$ the local ring $\left(R^{*},\left(s^{*}\right)\right)^{\wedge}$ does not satisfy $\mathcal{P}$.

Moreover, if $R$ is excellent (resp. Henselian) so is $R^{*}$.

Proof. The proof of Proposition 2.1 is an easy exercise, and also can be found in $[\mathrm{R}]$.

By using complete induction, we have seen, in [R], that certain (divisorial) properties of a local Noetherian ring remain stable under completion. This paper may serve as an example to illustrate that complete induction also can be used in the opposite direction, namely, to show that some properties of the completion descend to certain local Noetherian rings. As in $[R]$, the class of rings under consideration are rings with the complete approximation property. To explain this notion we first recall the definition of the approximation property by M. Artin:

2.2. Definition. Let $(R, m)$ be a local Noetherian ring, and $\hat{R}$ its $m$-adic completion. $R$ has the approximation property if every system of equations over $R$ which has a solution in $\hat{R}$ is already solvable in $R$. More precisely, let $X=\left(X_{1}, \ldots, X_{n}\right)$ be variables and let $f=\left(f_{1}, \ldots, f_{N}\right) \subseteq R[X]$ be an ideal in $R[X]$. Suppose that there is a solution $\hat{y}=\left(\hat{y}_{1}, \ldots, \hat{y}_{n}\right) \in \hat{R}^{n}$ of the system of equations $f=0$ in $\hat{R}$. Then there is an element $y=\left(y_{1}, \ldots, y_{n}\right) \in R^{n}$ with $f_{i}(y)=0$ for all $i=1, \ldots, N$.

Our aim is to show that local, Noetherian, Cohen-Macaulay rings with the approximation property inherit the canonical module from their completions. To apply complete induction in this situation, we want the approximation property to pass over to ideal-adic completions of the ring. These rings are called rings with the complete approximation property.

2.3. Definition. Let $(R, m)$ be a local Noetherian ring. We say that $R$ satisfies the complete approximation property if for every ideal $I \subseteq R$ the $I$-adic completion $(R, I)^{\wedge}$ of $R$ has the approximation property.

We believe that this is not truly a restriction on the class of rings with the approximation property. M. Artin conjectured that excellent, Henselian rings have the approximation property. As explained in $[R]$ it is a Corollary from Artin's conjecture that rings with the approximation property also have the complete approximation property. Artin's conjecture has already been shown for a large class 
of excellent, Henselian rings; for example, excellent and Henselian rings containing the rationals are known to possess the complete approximation property.

\section{The MAIN RESUlT}

This section is concerned with the proof of the following theorem:

3.1. Theorem. Let $(R, m)$ be a local Cohen-Macaulay ring with the complete approximation property. Then the canonical module $K_{R}$ of $R$ exists.

In order to prove the statement we first need the following well known result on the existence of the canonical module:

3.2. Theorem [HK, Satz 6.1 (a) $\Leftrightarrow(\mathrm{c})]$. Let $(R, m, k)$ be a local, Noetherian, Cohen-Macaulay ring of dimension $d$ and let $C$ be a finitely generated $R$-module. Then the following conditions are equivalent:

(a) $C \cong K_{R}$, i.e., the canonical module of $R$ exists and is isomorphic to $C$.

(b) $\operatorname{dim}_{k} \operatorname{Ext}_{R}^{i}(k, C)=\delta_{i d}$ for all integers $i \in \mathbb{N}$.

We are now ready to prove Theorem 3.1:

Proof. Suppose that there is a local Cohen-Macaulay ring $(R, m)$ with the complete approximation property for which the canonical module $K_{R}$ fails to exist. Then there is such a ring $(R, m)$ of minimal dimension $d$. Since local Artinian rings possess a canonical module, we obtain that $d>0$. We now proceed by complete induction on $R$. Since the completion $\hat{R}$ of $R$ admits a canonical module $K_{\hat{R}}$, by Proposition 2.1 there is an ideal $I \subseteq R$ such that:

(i) The canonical module of the $I$-adic completion $(R, I)^{\wedge}$ of $R$ does not exist.

(ii) For every element $s \in m(R, I)^{\wedge}-I(R, I)^{\wedge}$ the canonical module of $(R,(I, s))^{\wedge}$ exists.

By assumption $(R, I)^{\wedge}$ is also a ring with the complete approximation property and we may replace $R$ by $(R, I)^{\wedge}$. Thus we can assume that $R$ is a local CohenMacaulay ring with the approximation property for which the canonical module $K_{R}$ fails to exist. Moreover, there is a reduced nonmaximal ideal $I \subseteq R$ such that for every element $s \in m-I$ the canonical module of the $(s)$-adic completion $(R,(s))^{\wedge}$ exists. Pick an $R$-regular element $s \in m-I$ and let $\tilde{R}=(R,(s))^{\wedge}$. Let $\tilde{K}$ be the canonical module of $\tilde{R}$.

There is a short exact sequence of finitely generated $\tilde{R}$-modules

$$
0 \longrightarrow \tilde{L} \longrightarrow \tilde{R}^{n} \longrightarrow \tilde{K} \longrightarrow 0
$$

where we may consider $\tilde{L}$ as a submodule of the free module $\tilde{R}^{n}$.

(a) Claim: Suppose that for a positive integer $m \in \mathbb{N}$ there is an exact sequence of finitely generated $R$-modules

$$
0 \longrightarrow L \longrightarrow R^{n} \longrightarrow K \longrightarrow 0
$$

satisfying

$$
R^{n} /\left(L+s^{m} R^{n}\right) \cong \tilde{R}^{n} /\left(\tilde{L}+s^{m} \tilde{R}^{n}\right)
$$

as $R / s R$-modules (again $L$ is considered a submodule of $R^{n}$ ), and

$$
s \text { is a } K \text {-regular element. }
$$

Then $K$ is a canonical module of $R$. 
Proof of (a): We need to verify the condition in (b) of Theorem 3.2. Condition (a.1) implies that

$$
K / s K \cong \tilde{K} / s \tilde{K}
$$

as $R / s R$-modules. Since $s$ is a $\tilde{R}$-regular element, the module $\tilde{K} / s \tilde{K}$ is a canonical module of $R / s R$. We use criterion (b) in Theorem 3.2 together with Rees' Lemma [M, Lemma 2(i), p.140] to conclude that $K$ is a canonical $R$-module.

(b) To construct an exact sequence (**) which satisfies conditions (a.1) and (a.2) we consider part of a resolution of $\tilde{K}$,

$$
\tilde{R}^{t} \stackrel{\tilde{\varphi}}{\longrightarrow} \tilde{R}^{\ell} \stackrel{\tilde{\psi}}{\longrightarrow} \tilde{R}^{n} \longrightarrow \tilde{K} \longrightarrow 0
$$

where $\tilde{L}=\operatorname{im} \tilde{\psi}$. Since $s$ is $\tilde{R}$-regular, then $\operatorname{Tor}_{1}^{\tilde{R}}(\tilde{K}, \tilde{R} / s \tilde{R})=0$. Therefore the sequence (b.1) remains exact after tensoring with $\tilde{R} / s \tilde{R}$; i.e., the sequence

$$
\bar{R}^{t} \stackrel{\bar{\varphi}}{\longrightarrow} \bar{R}^{\ell} \stackrel{\bar{\psi}}{\longrightarrow} \bar{R}^{n} \longrightarrow \bar{K} \longrightarrow 0
$$

is exact where $\bar{R}=R / s R=\tilde{R} / s \tilde{R}$ and $\bar{K}=\tilde{K} / s \tilde{K}$. This shows that

$$
\left(\operatorname{im} \tilde{\varphi}+s \tilde{R}^{\ell}\right) / s \tilde{R}^{\ell}=\operatorname{im} \bar{\varphi}=\operatorname{ker} \bar{\psi}=\tilde{\psi}^{-1}\left(s \tilde{R}^{n}\right) / s \tilde{R}^{\ell} .
$$

We denote by $\tilde{V}$ the submodule $\tilde{\psi}^{-1}\left(s \tilde{R}^{n}\right)$ of $\tilde{R}^{\ell}$. Since $\tilde{V}$ contains $s \tilde{R}^{\ell}$, the $\tilde{R}$ module $\tilde{V}$ is extended from $R^{\ell}$; i.e., if we consider $R^{\ell}$ canonically as an $R$-submodule of $\tilde{R}^{\ell}$, the module $V=R^{\ell} \cap \tilde{V}$ extends to $\tilde{V}=V \otimes_{R} \tilde{R}$.

(c) We now suppose that there is a sequence of free $R$-modules and homomorphisms

$$
R^{t} \stackrel{\varphi}{\longrightarrow} R^{\ell} \stackrel{\psi}{\longrightarrow} R^{n}
$$

satisfying the following three conditions:

$$
\begin{aligned}
& \varphi \otimes_{R} \operatorname{id}_{\tilde{R}} \equiv \tilde{\varphi} \bmod s \tilde{R}^{\ell}, \\
& \psi \otimes_{R} \operatorname{id}_{\tilde{R}} \equiv \tilde{\psi} \bmod s \tilde{R}^{n} .
\end{aligned}
$$

This means that for all $\tilde{x} \in \tilde{R}^{t}$ we have $\left(\varphi \otimes_{R} \operatorname{id}_{\tilde{R}}\right)(\tilde{x})-\tilde{\varphi}(\tilde{x}) \in s \tilde{R}^{\ell}$ and, similarly, $\left(\psi \otimes_{R} \operatorname{id}_{\tilde{R}}\right)(\tilde{y})-\tilde{\psi}(\tilde{y}) \in s \tilde{R}^{n}$ for all $\tilde{y} \in \tilde{R}^{\ell}$.

$$
\operatorname{im} \varphi \subseteq \operatorname{ker} \psi \text {. }
$$

$$
\operatorname{im} \varphi+s R^{\ell}=V .
$$

Put $L=\operatorname{im} \psi$ and $K=R^{n} / \operatorname{im} \psi$. Our aim is to show that, under the above conditions, the exact sequence

$$
0 \longrightarrow L \longrightarrow R^{n} \longrightarrow K \longrightarrow 0
$$

satisfies conditions (a.1) and (a.2) (for $m=1$ ). In particular, $K$ is a canonical module of $R$. We first need to verify:

(d) Claim: Suppose conditions (c.1)-(c.4) hold. Then the sequence (c.1) is exact.

Proof of (d) Let $a=\left(a_{1}, \ldots, a_{\ell}\right) \in \operatorname{ker} \psi$. Then $\tilde{\psi}(a)=\tilde{\psi}(a)-\psi(a) \in s \tilde{R}^{n}$ by (c.2), implying that $a \in \tilde{\psi}^{-1}\left(s \tilde{R}^{n}\right) \cap R^{\ell}=V$. By (c.4) there are elements $b \in R^{t}$ and $c \in R^{\ell}$ with $a=\varphi(b)+s c$. Formula (c.3) implies that $s \psi(c)=0$ and thus $\psi(c)=0$ since the element $s$ is $R$-regular. This shows that $\operatorname{ker} \psi=\operatorname{im} \varphi+s \operatorname{ker} \psi$ and hence $\operatorname{ker} \psi=\operatorname{im} \varphi$. 
(e) The second congruence in (c.2) yields that $L \tilde{R}+s \tilde{R}^{n}=\tilde{L}+s \tilde{R}^{n}$, implying

$$
R^{n} /\left(L+s R^{n}\right) \cong \tilde{R}^{n} /\left(\tilde{L}+s \tilde{R}^{n}\right) .
$$

It remains to show that the element $s$ is also $K$-regular. To see this we tensorize the sequence (c.1) with $\bar{R}=R / s R$ and by (c.2) obtain the exact sequence

$$
\bar{R}^{t} \stackrel{\bar{\varphi}}{\longrightarrow} \bar{R}^{\ell} \stackrel{\bar{\psi}}{\longrightarrow} \bar{R}^{n} \longrightarrow \bar{K} \longrightarrow 0 .
$$

Therefore $\operatorname{Tor}_{1}^{R}(K, \bar{R})=0$ and hence $s$ is $K$-regular since $s$ is $R$-regular.

(f) Finally, it is an easy exercise, using the approximation property of $R$, to construct a sequence (c.1) which satisfies conditions (c.2), (c.3), and (c.4).

3.3. Remark. Complete induction allows us to replace the completion of $R$ by its completion with respect to the principal ideal $(s)$ of $R$. This maneuver allows us to establish, almost trivially, that $K$ is a canonical $R$-module. Without complete induction the proof of (a) seems much harder technically, if at all possible, following the above line of proof.

It is well known that in local Noetherian Cohen-Macaulay rings the existence of a canonical module is equivalent to the existence of a dualizing complex. Therefore:

3.4. Corollary. Let $(R, m)$ be a local Cohen-Macaulay ring with the complete approximation property. Then $R$ admits a dualizing complex.

\section{REFERENCES}

[A] M. Artin, Algebraic approximation of structures over complete local rings, Publ. Math. IHES, 36, (1969), 23-58. MR 42:3087

[H] V. Hinich, Rings with approximation property admit a dualizing complex, Math. Nachr. 163 (1993), 289-296. MR 94h:13017

[HK] F. Herzog and E. Kunz, Der kanonische Modul eines Cohen-Macaulay Rings, Lecture Notes in Math., vol. 238, Springer, New York, 1971.

[M] H. Matsumura, Commutative ring theory, Cambridge University Press, Cambridge, 1986. MR 88h:13001

[R] C. Rotthaus, Divisorial ascent in rings with the approximation property, J. of Algebra 178 (1995), 541-560.

Department of Mathematics, Michigan State University, East Lansing, Michigan 48824-1027

E-mail address: rotthaus@mth.msu.edu 